\title{
Bioengineered Internal Anal Sphincter Derived From Isolated Human Internal Anal Sphincter Smooth Muscle Cells
}

\author{
SITA SOMARA, * ROBERT R. GILMONT, ${ }^{*}$ ROBERT G. DENNIS, ${ }^{\ddagger}$ and KHALIL N. BITAR* \\ *Department of Pediatrics-Gastroenterology University of Michigan, Ann Arbor, Michigan; and ${ }^{\ddagger}$ Department of Biomedical Engineering, University of North Carolina, \\ Chapel Hill, North Carolina
}

BACKGROUND \& AIMS: The internal anal sphincter (IAS) is a specialized circular smooth muscle that maintains rectoanal continence. In vitro models are needed to study the pathophysiology of human IAS disorders. We bioengineered sphincteric rings from human IAS smooth muscle cells (SMC) and investigated their response to cholinergic stimulation as well as investigated whether protein kinase $\mathrm{C}(\mathrm{PKC})$ and Rho kinase signaling pathways remain functional. METHODS: 3-Dimensional bioengineered ring (3DBR) model of the human IAS was constructed from isolated human IAS SMC obtained from surgery. Contractile properties and force generation in response to acetylcholine, $\mathrm{PKC}$ inhibitor calphostin-C, Rho/ROCK inhibitor Y-27632, permeable Rho/ROCK inhibitor c3-exoenzyme, and PKC activator PdBU was measured. RESULTS: The human IAS 3DBR has the essential characteristics of physiologically functional IAS; it generated a spontaneous myogenic basal tone, and the constructs were able to relax in response to relaxants and contract in response to contractile agents. The constructs generated dose-dependent force in response to acetylcholine. Basal tone was significantly reduced by calphostin-C but not with Y-27632. Acetylcholine-induced force generation was also significantly reduced by calphostin-C but not with Y-27632. PdBU generated force that was equal in magnitude to acetylcholine. Thus, calphostin-C inhibited PdBU-induced force generation, whereas Y27632 and c3 exoenzyme did not. CONCLUSIONS: These data indicate that basal tone and acetylcholine-induced force generation depend on signaling through the PKC pathway in human IAS; PKC-mediated force generation is independent of the Rho/ ROCK pathway. This human IAS 3DBR model can be used to study the pathophysiology associated with IAS malfunctions.

ecal incontinence is a common clinical condition
that can have devastating social, psychological, and economic consequences for children and adults. Between $2 \%$ and $15 \%$ of the population suffers from fecal incontinence as assessed by epidemiologic studies. ${ }^{1,2}$ Many factors influence fecal continence in adults and children Stool consistency and volume, colonic transit time, and rectal compliance all place varying demands on the anal sphincters. ${ }^{3}$

Three structures maintain anal canal closure: the smooth muscle internal anal sphincter (IAS), the striated muscle external anal sphincter, and the striated muscle puborectalis muscle. ${ }^{4}$ The fatigue-resistant smooth muscle of the IAS provides $75 \%$ of closure pressure that is measured in the anal canal during normal activities. ${ }^{5}$ In biomechanical terms, this means that the smooth muscle generates sufficient inward radial stress that it constrains the outer diameter of the vascular mucosa to maintain constant apposition of opposing anal canal surfaces during normal activities. The smooth muscle is therefore the most important component in the maintenance of tone and thus continence. ${ }^{6}$ During times of increased need, when temporary increases in rectal pressure occur, activation of external anal sphincter and puborectalis augment the inward radial closure stress acting within the wall of the anus so as to maintain canal closure and prevent leakage. Fecal incontinence is associated with reduced anal closure pressure and mechanical stress maintaining tissue cooptation and closure of the anal canal. 7,8 This is largely attributable to decreased mechanical efficiency of the internal anal sphincter, but the specific causes may vary by age and injury. ${ }^{9-11}$

The IAS is a specialized circular smooth muscle with elevated basal tone as compared with the rectum, and it plays a significant role in rectoanal continence. The IAS is characterized by its ability to maintain tone and to relax, allowing the passage of feces. Although the major tone is due to the myogenic properties, a number of neurohumoral and hormonal responses of the muscle cells also cause the tone in the IAS. ${ }^{3,4}$

Abbreviations used in this paper: 3DBR, 3-dimensional bioengineered ring; IAS, internal anal sphincter; PKC, protein kinase C; SMC, smooth muscle cells.

(C) 2009 by the AGA Institute 0016-5085/09/\$36.00

doi:10.1053/j.gastro.2009.03.036 
IAS smooth muscles represent tonic muscles that remain contracted at rest and have small amplitude, slow contraction, and slow relaxation response, whereas nonIAS smooth muscle represents phasic muscle that shows a wide range of contractile activity varying from a fully relaxed basal state to a large-amplitude rapid contraction and rapid relaxation response. ${ }^{6}$ Although we know that the IAS plays a crucial role in the maintenance of anal continence, little is known about the cellular and biochemical mechanisms at work in the functional IAS. Smooth muscles can be categorized as "tonic" or "phasic" based on their mechanical and electrophysiologic characteristics. The IAS is an example of a truly tonic smooth muscle. ${ }^{12}$ The basal tone of the IAS is thought to be myogenic in nature. This means that the majority of the basal tone in the IAS is neither under the adrenergic influence of the sympathetic nervous system nor under the cholinergic control of the parasympathetic system. The basal tone is myogenic in nature because of specialized properties of the smooth muscle cells (SMC) of the IAS. ${ }^{13}$ Maintenance of MLC20 phosphorylation, which is an integral part of the myogenic control of sustained force generation, is regulated by at least 2 biochemical cascades: the protein kinase $\mathrm{C}(\mathrm{PKC})$ pathway ${ }^{14,15}$ and the Rho kinase pathway. ${ }^{16}$

Previous work using SMC harvested from rabbit IAS has shown that it is feasible to create functional physiologic ring models of the IAS and colon and that the IAS model generates spontaneous basal tone, dose-dependent contraction with cholinergic stimulation, and cAMP-dependent relaxation. ${ }^{17}$ We have for the first time bioengineered 3-dimensional (3D) rings from isolated SMC from human IAS. Our objective in this project was to determine the feasibility of bioengineering of IAS ring constructs from human IAS SMC and to test the hypothesis that the PKC and Rho Kinase pathways remain intact and functional in the human in vitro IAS bioengineered sphincteric rings. This will help us to develop more effective methods of treatment for this devastating condition.

\section{Materials and Methods}

Growth media consisted of Dulbecco's modified Eagle medium (DMEM) (catalog No. 12430 - 054; GIBCO, Invitrogen, Carlsbad, CA) with $15 \%$ fetal bovine serum (FBS), 3\% penicillin-streptomycin, and $0.6 \%$ L-glutamine. Differentiation media consisted of $73 \%$ DMEM (catalog No. 12430-054; GIBCO), 20\% media $199,7 \%$ heat-inactivated horse serum, and $1 \%$ penicillinstreptomycin. Hank's balanced salt solution (HBSS) contained $9.8 \mathrm{~g}$ HBSS and $0.35 \mathrm{~g} \mathrm{NaHCO} 3$ in $1 \mathrm{~L}$ distilled water. HEPES buffer ( $\mathrm{pH}$ 7.4) contained (in mmol/L) 115 $\mathrm{NaCl}, 5.7 \mathrm{KCl}, 2.0 \mathrm{KH}_{2} \mathrm{PO}_{4}, 24.6$ HEPES, $1.9 \mathrm{CaCl}_{2}, 0.6$ $\mathrm{MgCl}_{2}, 5.6$ glucose, $0.01 \%$ soybean trypsin inhibitor, and
0.184 (wt/vol) DMEM; $0 \mathrm{Ca}^{2+} / 2 \mathrm{mmol} / \mathrm{L}$ EGTA HEPES solution was the same except that $\mathrm{CaCl}_{2}$ was not included and $2 \mathrm{mmol} / \mathrm{L}$ EGTA was added.

\section{Cell Isolation}

SMC were isolated from the human IAS obtained after surgery from National Development and Research Institute (NDRI; New York, NY; project code: BIK1 001; protocol code: 001; IRBMED No. 1991-0297). The IAS tissue obtained was rapidly cleaned and stripped of connective tissue and striated muscle in ice-cold carbonated HBSS solution containing $3 \mathrm{X}$ penicillin-streptomycin and $2 \mathrm{X}$ antimycotic. The tissue was cut into small pieces and incubated in $15 \mathrm{~mL}$ HBSS buffer with $0.1 \%$ collagenase type II (Worthington Biochemical, Lakewood, NY) and $20 \mathrm{mg} / \mathrm{mL}$ DNAse- 1 for digestion at $37^{\circ} \mathrm{C}$ with $5 \%$ $\mathrm{CO}_{2}$ for a 1-hour digestion period. Fresh HBSS $(15 \mathrm{~mL})$ buffer with collagenase was added, and the contents were mechanically dissociated using a $10-\mathrm{mL}$ pipette and incubated for 1 additional hour. After the second digestion period was complete, the cells were collected and centrifuged at $600 \mathrm{~g}$ for 5 minutes, and the supernatant was discarded. The cells were washed and resuspended 3 times to ensure removal of excess collagenase and then resuspended in DMEM.

\section{In Vitro 3D Human IAS Model Bioengineering From Human IAS SMC}

Culture plates $(35 \mathrm{~mm})$ were prepared as described previously. ${ }^{18-20}$ Human IAS was obtained after surgery from NDRI. Primary SMC were isolated as described previously ${ }^{14}$ and cultured in $75-\mathrm{cm}^{2}$ flasks. Once confluent, cells $(\sim 100 \mathrm{~K})$ were transferred to plates containing a loose fibrin gel on which they proliferated to confluence. As cells proliferated, they shrunk the fibrin gel, contracting it toward the center of the culture dish around a 5-mm-diameter SYLGARD (World Precision Instruments, Sarasota, FL) mold. ${ }^{20}$ Cells migrated and self-assembled along the line of force until they formed a parallel array of cells. Tissues were considered "formed" when a 3D cylindrical tube of sphincteric tissue contracted around the SYLGARD mold. ${ }^{20}$ All fibrin constructs seeded with IAS cells were fully formed within 5-10 days after cell seeding. The resulting IAS 3-dimensional bioengineered ring (3DBR) constructs remained stable in culture and were experimentally tested.

\section{Measurement of Contractile Properties and Force Generation}

The protocol for measuring excitability and contractility of engineered muscle constructs was adapted from previous work. ${ }^{18,19}$ Briefly, human IAS 3DBR were separated from their molds using forceps, and the minimum ring diameter was measured using a calibrated eyepiece and a $5 \times$ or $10 \times$ objective lens on an inverted 
microscope (Axiovert 25; Zeiss, Thornwood, NY). The dish was placed on a heated aluminum platform that was maintained at a temperature of $37^{\circ} \mathrm{C}$ until the testing was complete. For measurements of force generation, one end of the engineered IAS was anchored by a stainless steel pin $(10 \mathrm{~mm} \times 0.1 \mathrm{~mm}$ diameter $)$ to the SYLGARD substrate, and another stainless steel pin was bent in the shape of a hook and attached by canning wax to an optical force transducer with a resolution of $1.4 \mu \mathrm{N}$ and a range of $2 \mathrm{mN} \cdot$.8,19,21 $^{2}$ Spontaneous basal tone of IAS muscle constructs was measured by stretching of the tissue $(50 \%)$ using a 3 -axis micromanipulator, followed by a period of equilibrium (between 20 and 60 minutes), where the IAS rings stabilized, resulting in the establishment of a new stable baseline of tension. ${ }^{22,23}$

\section{Testing Protocol}

Protocols were designed to confirm that bioengineered IAS rings exhibited parameters similar to those of physiologically functional IAS. Bioengineered IAS rings were stretched $50 \%$ of their resting length using a 3 -axis micromanipulator and were allowed to sit for 20-30 minutes to reestablish a stable baseline. The development of basal tone was analyzed. Relaxation of basal tone was studied by adding 8-br-cAMP after stable basal tone was generated. Bioengineered IAS rings were treated with $\left(10^{-10}-10^{-6} \mathrm{~mol} / \mathrm{L}\right)$ acetylcholine (Ach) to study dosedependent force generation. Relaxation of Ach-induced force generation was studied by adding $10^{-6} \mathrm{~mol} / \mathrm{L}$ 8-brcAMP. Bioengineered IAS rings were further tested to study if they were capable of regenerating force postrelaxation. Force generation in response to PKC activator PdBU was also studied. The testing protocol described was then repeated once in the presence of $10^{-6} \mathrm{~mol} / \mathrm{L}$ calphostin C, an inhibitor of PKC, and repeated again in the presence of Y-27632, a selective inhibitor of Rhoassociated protein kinase.

\section{SDS-PAGE and Western Blotting}

For 1-dimensional SDS-PAGE, the samples were mixed in an equal volume of $2 \mathrm{X}$ sample buffer $(50$ $\mathrm{mmol} / \mathrm{L} \mathrm{Tris,} 10 \%$ [vol/vol] glycerol, $2 \%$ [wt/vol] SDS, and $0.1 \%$ [wt/vol] bromophenol blue, $\mathrm{pH}$ 6.8). The proteins from whole cell lysates of human IAS SMC and human colon SMC were separated by $10 \%$ or $12 \%$ SDS-PAGE and transferred onto nitrocellulose or polyvinylidene difluoride membranes. The polyvinylidene difluoride membrane was then blocked with 5\% nonfat dry milk for 1 hour. Following blocking, the membrane was incubated in an appropriate dilution of primary antibody in 5\% nonfat dry milk in Tris-buffered saline Tween-20 (TBST) for 1 hour. The membrane was washed thrice with TBST to remove unbound primary antibody for 15 minutes each wash at room temperature. The membrane was then incubated in an appropriate dilution of secondary anti- body in 5\% nonfat dry milk in TBST for 1 hour at room temperature. The membrane was washed 3 times with TBST for 15 minutes each wash at room temperature to remove unbound secondary antibody. The membrane was then incubated with ECL (Enhanced chemiluminescence) reagent for 1 minute. The proteins were detected on the membrane by immediately exposing the membrane to the film for 30 seconds and 1 minute.

\section{Isolation of Detergent-Resistant Membrane Rafts}

Detergent-resistant membrane micro-domains (lipid rafts) were isolated essentially as described. ${ }^{24}$ Briefly, cultured SMC were collected by scraping and recovered by centrifugation before being homogenized in ice-cold MES (2-[ $N$-morpholino]ethanosulfonic acid)-buffered saline containing $1 \%$ Triton $\mathrm{X}-100$. The homogenate composition was made of $40 \%$ sucrose with a volume of 400 $\mu \mathrm{L}$ and transferred to an ultracentrifuge tube. The homogenate was sequentially over-layered with $900 \mu \mathrm{L}$ of $30 \%$ and $900 \mu \mathrm{L}$ of $5 \%$ sucrose solutions and centrifuged for 20 hours at $\sim 250,000 \mathrm{~g}(54000 \mathrm{rpm})$. Following centrifugation, $200-\mu \mathrm{L}$ fractions were serially removed from tube top to bottom, the pellet was suspended in $200 \mu \mathrm{L}$ RIPA buffer by sonication, and the fractions were subjected to SDS-PAGE and immunoblot analysis. The presence of caveolin in fractions taken from the 5\% sucrose $30 \%$ sucrose interface (fraction 5-7) indicate the successful isolation of lipid rafts.

\section{Statistical Analysis}

All force measurements were collected at the rate of 100 samples/second for 600 seconds and recorded using a computer with LabVIEW data acquisition software (National Instruments, Austin, TX). Force was determined by subtracting the baseline from the total force values. Data analysis was done using LabVIEW and Microsoft Excel (Microsoft Corp, Redmond, WA) software programs. For studies with the force graphs, random peaks were picked within a defined period of time from 3 to 5 sets of experiments performed on bioengineered human IAS 3DBR constructs. Results from different treatments to human IAS 3DBR were expressed as means \pm SEM. An unpaired 2-tailed Student $t$ test was used to compare differences among groups.

\section{Results}

\section{Development of Spontaneous Basal Tone in Human 3D-Bioengineered IAS Rings}

Stretching of an IAS ring by $50 \%$ of its length resulted in the development of spontaneous basal tone (Figure 1A). The human IAS 3DBR generated a basal tone of $11.33 \pm 0.88 \mu \mathrm{N}(\mathrm{n}=3)$ of force. Upon preincubation with the PKC inhibitor calphostin $\mathrm{C}$ for 20 minutes, a 

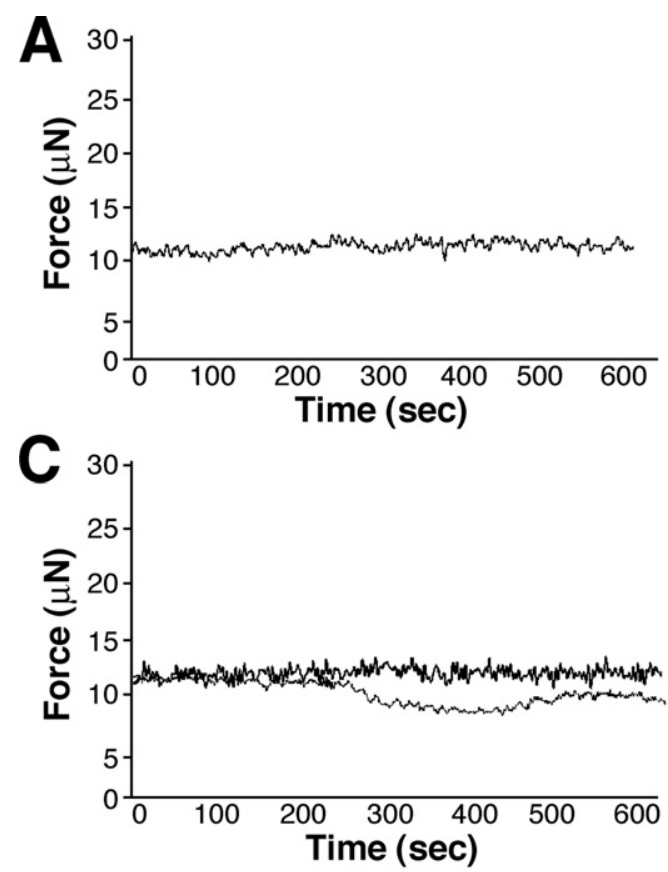

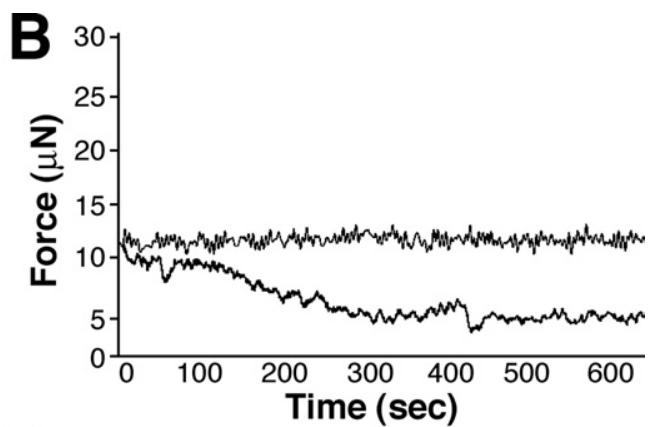

D

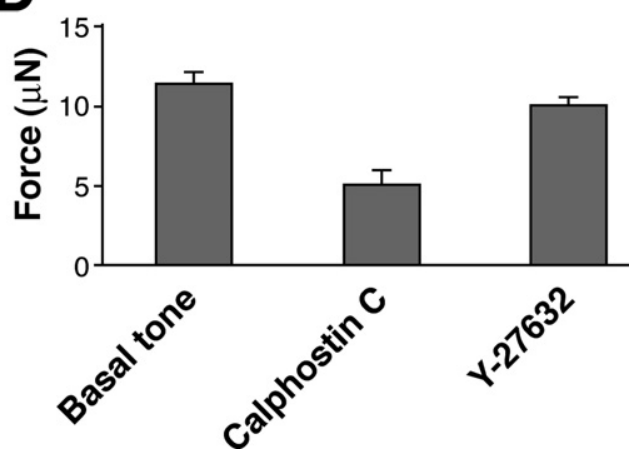

Figure 1. Basal force generation in bioengineered human IAS. (A) Human IAS 3DBR generated a spontaneous basa tone. (B) A significant and sustained decrease in basal tone was observed upon incubation with the PKC inhibitor calphostin C (Calph-C). This decrease was characterized by an attenuation of the magnitude of basal force generated and an immediate relaxation of basal tone. (C) A small transient decrease was observed in the basal tone upon incubation with the ROCK inhibitor Y-27632. This decrease was characterized by a delayed attenuation of smaller magnitude of basal tone. $(D)$ Graphic representation of the basal tone and effect of inhibitors on the basal tone. Note that calphostin-C was more effective at reducing the IAS basal tone. significant reduction in the basal tone $(5.00 \pm 1.00 \mu \mathrm{N}$; $\mathrm{n}=3 ; P \leq .5$ ) was observed (Figure $1 B$ ). Inhibition of PKC was immediate, and relaxation of basal tone was sustained. Preincubation with Rho/ROCK inhibitor Y-27632 did not show any significant change in the basal tone $(10.00 \pm 0.58 \mu \mathrm{N} ; \mathrm{n}=3 ; P \leq .5)$ (Figure $1 C$ ). Inhibition of RhoA was delayed and was of smaller magnitude than that induced by PKC inhibitor indicating an earlier and greater role of PKC without excluding a delayed role for RhoA pathway. The data suggest that both PKC and RhoA pathways contribute to the development of basal tone but may be at different levels (time points, percentage) and that their effect could be overlapping in time and magnitude. ${ }^{13,25} \mathrm{~A}$ graph representing the data showing the role of PKC and RhoA pathway in the generation of the basal tone is shown (Figure 1D).

\section{Acetylcholine-Induced Contractile Response of Human 3D-Bioengineered IAS Rings}

Human 3D-bioengineered IAS rings responded to the contractile neurotransmitter Ach and generated force (Figure $2 A)$. Ach induced a dose-dependent $\left(10^{-10}\right.$ $\left.10^{-6} \mathrm{~mol} / \mathrm{L}\right)$ force generation with Ach $\left(10^{-6} \mathrm{~mol} / \mathrm{L}\right)$ inducing maximal sustained force (up to 5 minutes) (27.67 $\pm 1.73 \mu \mathrm{N} ; \mathrm{n}=4 \mathrm{ie}, \sim 16 \mu \mathrm{N}$ above baseline) (Figure $2 A$ and $2 B$ ).

\section{Role of RhoA and PKC Pathway on Contractile Response of Human IAS 3DBR}

Upon preincubation with the PKC inhibitor calphostin $\mathrm{C}$, a significant reduction $(9.00 \pm 2.00 \mu \mathrm{N} ; \mathrm{n}=$ $2 ; P \leq .5)$ in Ach-induced force generation was observed (Figure 3A). Preincubation with Rho/ROCK inhibitor
Figure 2. Ach-induced force generation in bioengineered human IAS. (A) A rapid and sustained increase in force generation in response to acetylcholine $\left(10^{-6} \mathrm{~mol} / \mathrm{L}\right)$ was observed in bioengineered human IAS. (B) A dose-dependent increase in force generation was observed in response to increasing concentrations of acetylcholine $\left(10^{-10}-0^{-6} \mathrm{~mol} / \mathrm{L}\right)$.
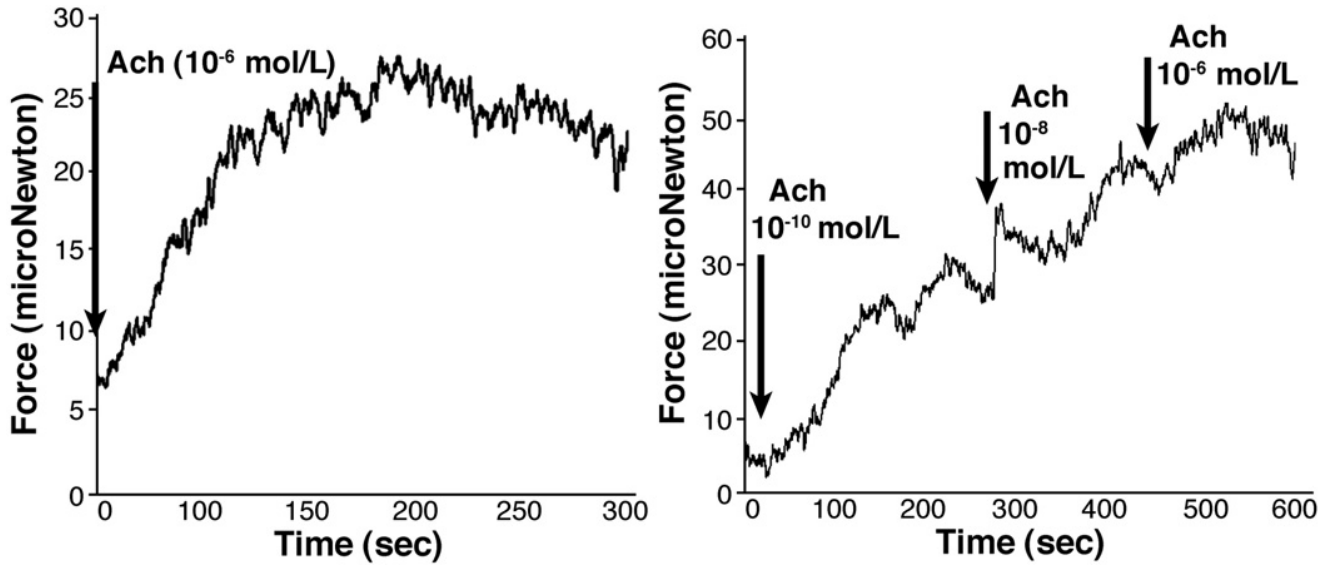
Figure 3. Effect of inhibitors of RhoA and PKC pathway on contractile response of human IAS 3DBR. (A) Preincubation with Rho/ROCK inhibitor Y-27632 induced a latent onset with decrease in the maximal Achinduced force generation. (B) Preincubation with Rho/ROCK inhibitor C3-exoenzyme also induced a latent onset, with decrease in the maximal Achinduced force generation. (C) Preincubation with PKC inhibitor calphostin $\mathrm{C}$ showed a significant reduction in the Ach-induced force generation. (D) Graphic representation of the Ach-induced force generation and the effect of inhibitors on the Ach-induced force generation. Note that calphostin-C showed the maximum decrease in Ach-induced force generation of human IAS 3DBR.

Y-27632 did show reduction $(18.50 \pm 2.08 \mu \mathrm{N} ; \mathrm{n}=3$; $P \leq .5$ ) in Ach-induced force generation (Figure $3 B$ ) but of lesser magnitude than compared with PKC inhibition, yet it showed a definite slowing in the onset of the response. Similarly, preincubation with c3-exoenzyme (permeable Rho/ROCK inhibitor) also showed reduction $(16.50 \pm 2.08 \mu \mathrm{N} ; \mathrm{n}=3 ; P \leq .5)$ in Ach-induced force generation and slowing of the onset of the response (Figure 3C). Graph represents the data showing the role of PKC and RhoA pathway in the generation of the force in response to Ach (Figure 3D). Data indicate role for both PKC and RhoA in the generation of force in IAS constructs, with a predominant role for PKC in the onset and a role for both PKC and RhoA in the maintenance of force generation.

\section{PdBU-Induced Contractile Response of Human 3D-Bioengineered IAS Rings}

Because inhibition of PKC showed significant and predominant effect on basal tone as well as on Achinduced force generation, we studied direct activation of PKC pathway on force generation in these bioengineered constructs. Stimulation of human IAS 3DBR constructs with PdBU, an activator of the PKC pathway, generated

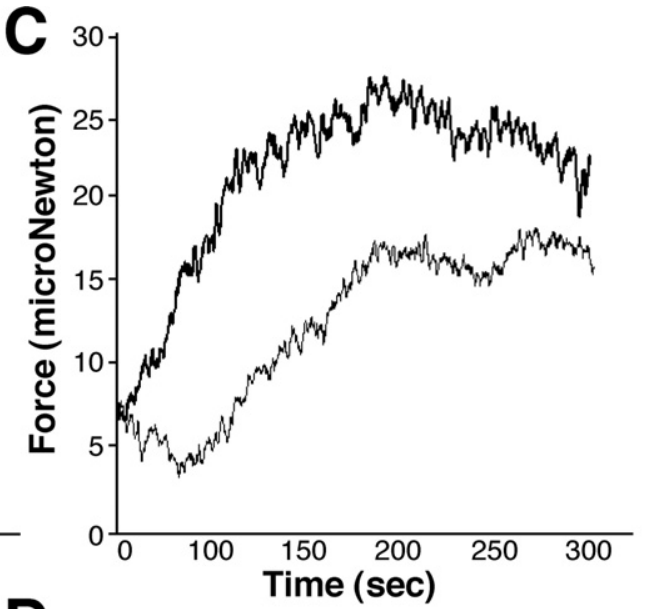

D

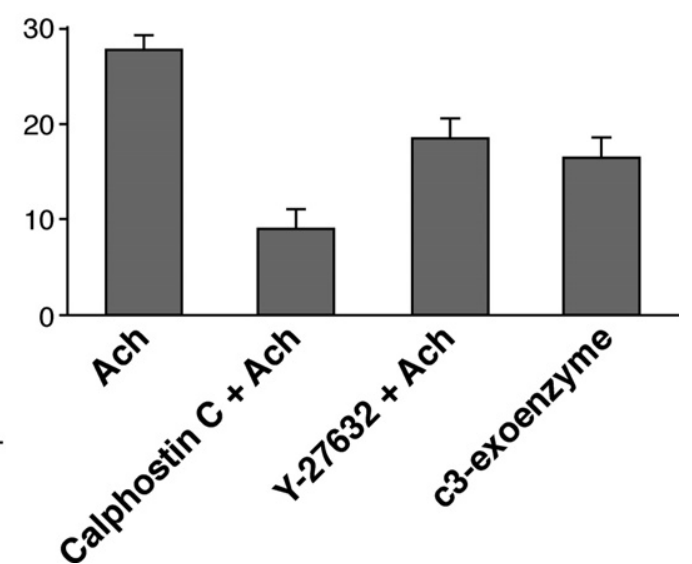

force of equal magnitude to the force generated upon Ach stimulation. The maximal sustained force generated by PdBU was $25.00 \pm 4.64 ; \mathrm{n}=3$ (ie, $\sim 14 \mu \mathrm{N}$ above baseline) (Figure 4). Upon preincubation with the PKC

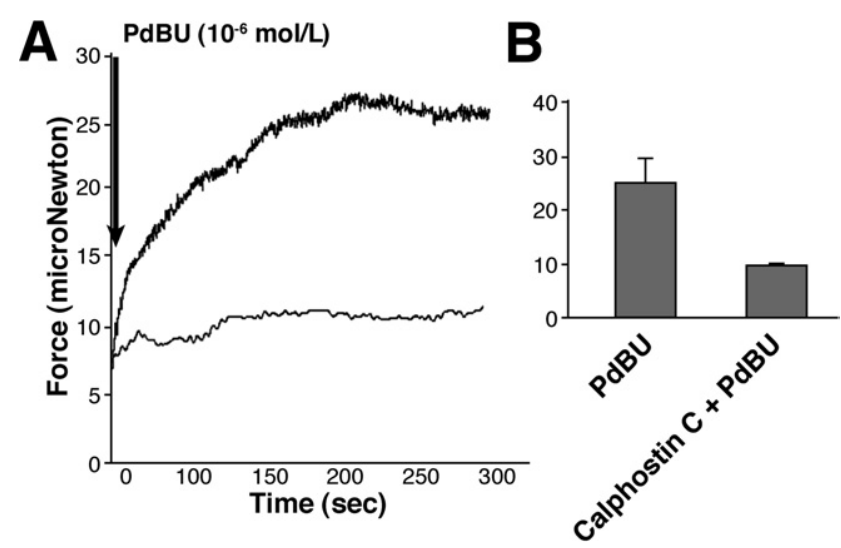

Figure 4. Effect of PKC inhibitor, calphostin $\mathrm{C}$ on $\mathrm{PdBU}$-induced force generation of human IAS 3DBR. (A) Preincubation with PKC inhibitor calphostin $\mathrm{C}$ showed a significant reduction in the PdBU-generated force. $(B)$ Graphic representation of the PdBU-induced force generation and the effect of PKC inhibitor calphostin $\mathrm{C}$ on the PdBU-induced force generation. 


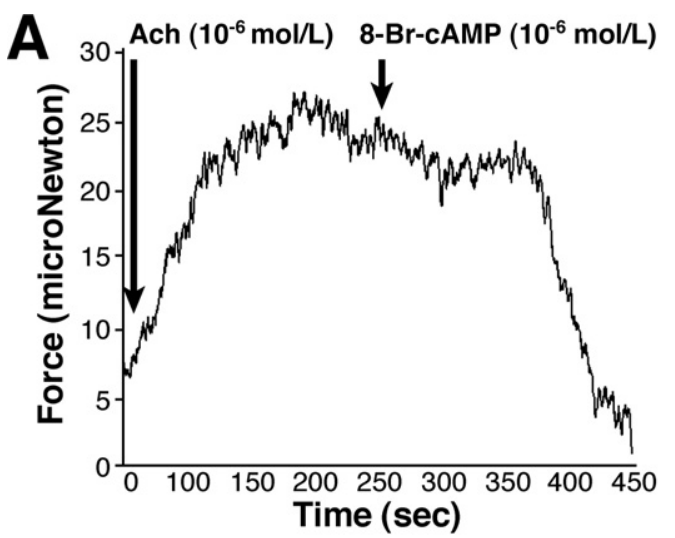

inhibitor, calphostin $\mathrm{C}$, a significant reduction (9.50 \pm $0.50 \mu \mathrm{N} ; \mathrm{n}=2 ; P \leq .05)$ in the PdBU-generated force was observed (Figure 4). Graph represents the data showing the effect of calphostin $\mathrm{C}$, a PKC inhibitor on force generation in response to PdBU (Figure 4B).

\section{cAMP-Induced Relaxation of Human 3D-Bioengineered IAS Rings}

In response to 8 -Br-cAMP $\left(10^{-6} \mathrm{~mol} / \mathrm{L}\right)$, there was reduction in force generation. Ach-induced contracted human IAS 3DBR demonstrated a sustained relaxation response reaching maximal relaxation after incubation with 8-Br-cAMP and subsequently resulting in the reestablishment of a new, stable basal tone. Average force reduction for the rings incubation with 8 -Br-cAMP was $21.50 \pm 2.50 \mu \mathrm{N} ; \mathrm{n}=2 ; P \leq .5$ (Figure $5 A$ ). Contraction of IAS 3DBR postrelaxation was studied by stimulating the relaxed IAS ring with $\mathrm{PdBU}$. PdBU stimulation of 8-Br-c-AMP-mediated relaxed human IAS 3DBR induced subsequent increase in force generation with a magnitude of $11.00 \pm 2.02 \mu \mathrm{N} ; \mathrm{n}=2 ; P \leq .5$ (Figure $5 B$ ).

\section{Biochemical Studies on Isolated Human IAS SMC}

Expression profile of human IAS cells vs circular colon smooth muscle in the basal resting state. Whole cell lysates of human IAS SMC and human colonic SMC were equally loaded and probed with specific monoclonal antibodies for each of the proteins (PKC $\alpha$, RhoA, CPI-17, and HSP27). An increased expression of $\operatorname{PKC} \alpha$, RhoA, CPI-17, and HSP27 was observed in human IAS SMC as compared with human colonic circular SMC at rest (Figure $6 A$ ).

Expression of caldesmon in human SMC from cultured colon and IAS. Whole cell lysates of confluent human IAS SMC and human colonic SMC in culture were equally loaded and probed with anticaldesmon antibody. An increased expression of caldesmon was observed in human IAS SMC as compared with human colonic circular SMC at rest (Figure 6B).
Figure 5. CAMP-induced relaxation of human 3D-bioengineered IAS rings. $(A)$ In response to 8-Br-cAMP, a dramatic decrease in Ach-induced force generation was observed suggesting relaxation of human IAS 3DBR. (B) Addition of PdBU to 8-Br-cAMP-relaxed human IAS 3DBR showed a restitution of force generation.

\section{Time (sec)}

Sequestration levels of PKC $\alpha$, RhoA, and HSP27 in caveolae of human IAS and human colonic circular SMC in the basal resting state. Caveolin-enriched membrane fractions of human IAS and colonic circular SMC were purified by sucrose density gradient method, and aliquots from each of 12 fractions were immunoblotted with either anti-cav-1 antibody or anti-PKC $\alpha$ antibody or anti-HSP27 or anti-RhoA antibody. Fractions 5, 6, 7 are caveolin-1-positive fractions. An increased sequestration level of PKC $\alpha$, RhoA, and HSP27 was observed in caveolae of human IAS as compared with human colonic SMC (Figure 7).

Bioengineering of 3D-IAS ring construct over 3 weeks of culture. 3D-ring constructs were observed under microscope over a period of 3 weeks to study the alignment and orientation of cells into 3D ring (Figure 8).

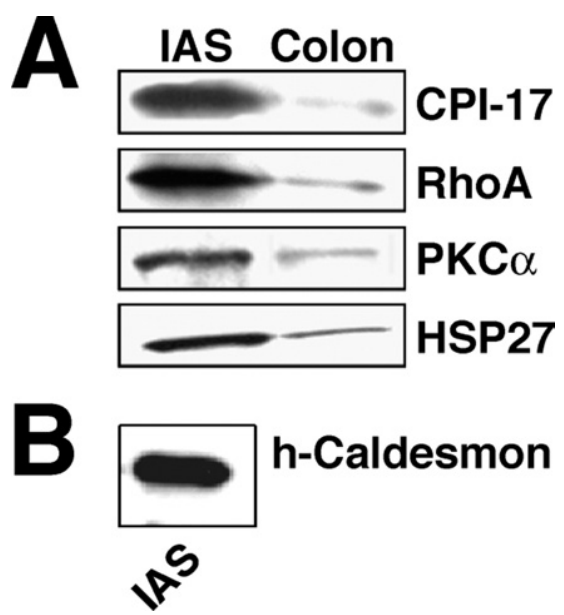

Figure 6. (A) Expression Profile of human IAS cells vs circular colon smooth muscle: Immunoblotting of whole cell lysate of confluent smooth muscle cells in culture from human IAS as compared with colonic circular muscle showed an increased expression of $\mathrm{PKC} \alpha$, RhoA, CPI-17 and HSP27 at rest. (B) Expression profile of h-caldesmon, a smooth muscle cell marker, in smooth muscle cells of human IAS: Immunoblotting of whole cell lysate of cultured confluent smooth muscle cells from human IAS showed a very distinct expression of smooth muscle marker h-caldesmon at rest. 


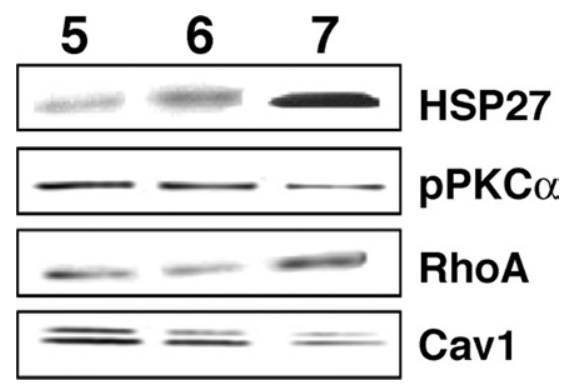

Figure 7. Sequestration of PKC $\alpha$, RhoA, and HSP27 in caveolae of human IAS smooth muscle cells in the basal resting state. Immunoblotting of caveolin-enriched membrane lipid raft fractions (fraction 5-6) from human IAS smooth muscle cells showed sequestration of PKC $\alpha$, HSP27, and RhoA in these fractions indicating preponderance of signaling molecules in the specific microdomains of the IAS smooth muscle cells membrane at rest.

Over a period of 3 weeks, the IAS SMC differentiated and align around the center sylgard post forming a $3 \mathrm{D}$ ring.

\section{Discussion}

Despite the frequency of fecal incontinence, little is known about the mechanisms underlying sphincter malfunction. Previous research tools to understand IAS physiology, including tissue culture, ${ }^{26}$ cell suspensions, ${ }^{22,27,28}$ and tissue explants, ${ }^{23,29,30}$ have their own limitations. New technologic advances in tissue engineering, cell survival, and utilization of 3D matrixes offered new opportunities to engineer 3D IAS sphincters. We have previously reported a successful construction of 3DBR from rabbit IAS SMC. Tissue engineering utilizes tissue culture techniques to provide a controlled environment with physiologic conditions for tissues to survive for extended periods of time. In the present study, for the first time, we have demonstrated the feasibility of bioengineering an in vitro $3 \mathrm{D}$ fibrin-based model of the human IAS. This technique uses a homogenous population of isolated human sphincteric SMC seeded on a fibrin gel. Upon seeding, cells began digesting the fibrin matrix, replacing it with their own extracellular matrix (ECM). ${ }^{31,32}$ On fibrin gels, the cells proliferate and selforganize along the line of force forming $3 \mathrm{D}$ functional tissues around the mold. ${ }^{33-37}$ The human IAS 3DBR formed in 5-10 days similar to our previous studies on rabbit IAS $3 \mathrm{DBR}^{20}$ and were successfully tested between 9 and 23 days providing evidence for viability beyond 3 weeks.

The bioengineered human IAS 3DBR exhibited physiologic behavior functionally similar to in vivo IAS. Human IAS 3DBR construct is able to respond to different neurotransmitters in vitro and is able to generate substantial force, which could be recorded. Our data show that human IAS 3DBR demonstrate stable, spontaneous basal tone (Figure 1A). Ach generated a rapid and significant force in IAS 3DBR (contraction) (Figure 2A), and, upon the addition of 8-Br-cAMP, IAS rings generated force decreased (relaxation) (Figure 5A). These data provide evidence of physiologic functionality of in vitro bioengineered human IAS construct. As expected, bioengineered IAS rings did not exhibit the spontaneous phasic contractile behavior exhibited by colonic rings at any time demonstrating tissue specificity. This suggests that IAS constructs exhibited physiologic functionality and characteristic tissue specificity that are unique to the IAS.

Basal tone generated by human IAS 3DBR had a magnitude of $11.3 \mu \mathrm{N}$. Upon stimulation with the same concentration of Ach, both human IAS 3DBR and isolated human IAS single cells exhibited peak contraction within 120 seconds after stimulation that was sustained for 4 minutes (Figure 2A). These similarities suggest that bioengineered IAS rings have the sensitivity of isolated single cells, which may be because of the lack the barriers present in muscle strips, such as innervating nerves, epithelial lining, and serosa, and therefore provide an in vitro opportunity to experimentally manipulate one $3 \mathrm{D}$ tissue constituent. Ach-induced contraction of both isolated single cells and bioengineered rings corresponds to previously described Ach-induced contraction of human IAS muscle strips. ${ }^{25,31,38}$ Human IAS 3 DBR also responded to PdBU, a PKC activator and generated a force of $25-\mu \mathrm{N}$ magnitude. Agonist-induced stimulation showed that human IAS 3DBR were functionally similar to IAS in vivo. Human IAS rings showed sustained relaxation of the basal tone in response to 8-Br-cAMP $\left(10^{-5}\right.$ $\mathrm{mol} / \mathrm{L}$ ). This is similar to IAS in vivo, where relaxation is

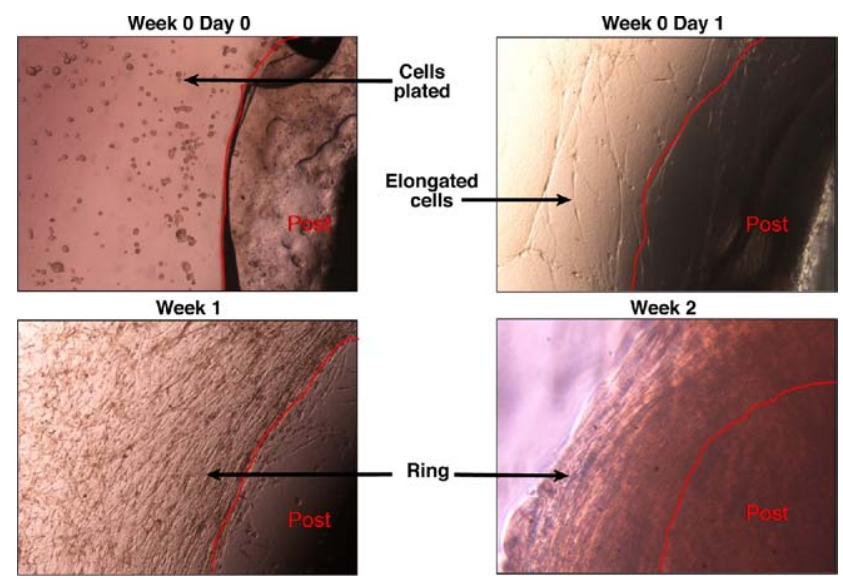

Figure 8. Bioengineering of 3D-IAS ring construct over 3 weeks of culture. Images of human IAS 3DBR construct were taken with Nikon Digital DS Fi1camera in real time while monitoring under inverted microscope. Week 0: image showing the cell plated on the fibrin gel around the center sylgard post. Week 0, day 1: Differentiated cells are beginning to elongate and adhere to the fibrin gel. Week 1: Differentiated smooth muscle cells closer to the post have started to align around the post while the cells in the periphery are in the process of alignment. Week 2: Differentiated smooth muscle cells have nicely aligned and oriented around the post. 
largely mediated by cAMP and is calcium independent. ${ }^{15,39-41}$ Addition of 8-Br-cAMP also induced rapid relaxation of Ach-induced contracted IAS rings (Figure 5). Preincubation of bioengineered human IAS 3DBR with Ach $\left(10^{-6} \mathrm{~mol} / \mathrm{L}\right)$ did not affect the relaxation response induced subsequently with 8 -Br-cAMP $\left(10^{-5}\right.$ $\mathrm{mol} / \mathrm{L}$ ) because IAS 3DBR demonstrated an immediate shift from contraction to relaxation after 8-Br-cAMPinduced stimulation (Figure 5A). Similarly, preincubation of human IAS 3DBR with PdBU did not affect the subsequent relaxation induced by 8-Br-cAMP. Furthermore, stimulation of 8-Br-cAMP-induced relaxed human IAS 3DBR with Ach- or PdBU (Figure 5B)-induced contraction. This suggests that bioengineered human IAS 3DBR have the ability to contract and relax within minutes, similar to the capacity seen in vivo. Therefore, human IAS 3DBR constructs exhibited several physiologically functional behaviors similar to the capacity seen in vivo.

Comparison of contractile protein expression profile of human IAS SMC with human circular colon SMC showed an increased expression of PKC $\alpha$, RhoA, CPI-17, and HSP27 in human IAS SMC as compared with human colonic circular SMC at rest. This could be the reason for the specificity of basal tone generated by IAS sphincteric smooth muscle. Further confirmation was done by analysis of caveolin-enriched membrane fractions of human IAS SMC. Immunoblotting analysis showed sequestration of PKC $\alpha$, RhoA, and HSP27 in membrane fractions $5,6,7$ that corresponds with the presence of caveolin indicating sequestration of $\mathrm{PKC} \alpha$, RhoA, and HSP27 in caveolae at rest.

The novel in vitro human 3DBR demonstrated a spontaneous basal tone and maintained the biochemical pathways crucial to the development of sphincteric tone (PKC and Rho kinase). Rattan et al have recently demonstrated the importance of the Rho kinase pathway in the development of basal tone of the internal anal sphincter. ${ }^{27}$ Present data show that inhibition of Rho kinase pathway by Y-27632 does inhibit the basal tone in human IAS 3DBR, confirming the response of 3DBR to match the in vivo IAS. Furthermore, Rattan et $\mathrm{al}^{27}$ state that "PKC has been speculated to play a role in the tonic contraction of certain smooth muscles; however, its exact role in the basal tone of the IAS is not known." These studies demonstrated that the PKC pathway is intact in in vitro human IAS 3DBR models, as evidenced by the force generated in the presence of PdBU, a PKC pathway activator. Our present data suggest that PKC likely plays an important role in the onset and maintenance of the basal tone of the IAS. In addition, these studies show that Rho Kinase and PKC pathways function independently. The response to PdBU was effectively blocked by calphostin $\mathrm{C}$, an inhibitor of the PKC pathway. This blocked re- sponse was not seen in the presence of Y-28376, an inhibitor of the Rho kinase pathway. Because calphostin $\mathrm{C}$ did lower the basal level of basal tone, it could be that calphostin $\mathrm{C}$ inhibited basal tone as well as it did inhibit the response to stimulation by $\mathrm{PdBU}$, which was expected because PdBU specifically stimulated PKC.

These studies evaluate for the first time the methods and feasibility of generating a functional fibrin-based bioengineered model of the human IAS derived from human cells. The resulting human IAS 3DBR constructs show tissue specificity to the IAS, are highly reproducible, and are functionally similar to IAS smooth muscle in vivo. Our fibrin-based constructs provide the opportunity to test the effects of various pharmacologic agents, growth factors, and mechanical interventions on smooth muscle function. This is the first report of a functional in vitro model of human IAS that may be used in the elucidation of the mechanisms causing smooth muscle sphincter myogenic malfunction and the investigation of treatments for fecal incontinence.

\section{References}

1. Drossman DA, Li Z, Andruzzi E, et al. US householder survey of functional gastrointestinal disorders. Prevalence, sociodemography, and health impact. Dig Dis Sci 1993;38:1569-1580.

2. Perry S, Shaw C, McGrother C, et al, and Leicestershire MRC Incontinence Study Team. Prevalence of faecal incontinence in adults aged 40 years or more living in the community. Gut 2002 50:480-484.

3. Rao SSC, Kempf J, Stessman M. Anal seepage:sphincter dysfunction or incomplete evacuation (abstr)? Gastroenterology 1998;114:A824.

4. Bharucha AE. Fecal incontinence. Gastroenterology 2003;124: 1672-1685.

5. Frenckner B. Function of the anal sphincters in spinal man. Gut 1975;16:638-644

6. Sahai E, Olson MF, Marshall CJ. Cross-talk between Ras and Rho signalling pathways in transformation favours proliferation and increased motility. EMBO J 2001;20:755-766.

7. Sun WM, Read NW, Donnelly TC. Impaired internal anal sphincter in a subgroup of patients with idiopathic fecal incontinence. Gastroenterology 1989;97:130-135.

8. Delechenaut P, Leroi AM, Weber J, et al. Relationship between clinical symptoms of anal incontinence and the results of anorectal manometry. Dis Colon Rectum 1992;35:847-849.

9. Sultan AH, Kamm MA, Hudson CN, et al. Third degree obstetric anal sphincter tears: risk factors and outcome of primary repair. Br Med J 1994;308:887-891.

10. Laurberg S, Swash M. Effects of aging on the anorectal sphincters and their innervation. Dis Colon Rectum 1989;32:737-742.

11. Jones OM, Brading AF, Mortensen NJ. The physiology, pharmacology and therapeutic manipulation of the internal anal sphincter. Can J Physiol Pharmacol 2002;16:249-257.

12. Szymanski PT, Szymanska G, Goyal RK. Differences in calmodulin and calmodulin-binding proteins in phasic and tonic smooth muscles. Am J Physiol Cell Physiol 2002;282:C94-C104.

13. Rattan S, Regan RF, Patel CA, et al. Nitric oxide not carbon monoxide mediates nonadrenergic noncholinergic relaxation in the murine internal anal sphincter. Gastroenterology 2005;129: 1954-1966. 
14. Bitar KN, Hillemeier C, Biancani P, et al. Regulation of smooth muscle contraction in rabbit internal anal sphincter by protein kinase C and Ins(1,4,5)P3. Am J Physiol 1991;260:G537-G542.

15. Chakder S, Rattan S. Involvement of CAMP and cGMP in relaxation of internal anal sphincter by neural stimulation, VIP, and NO. Am J Physiol 1993;264:G702-707.

16. Patil SB, Bitar KN. RhoA- and PKC- $\alpha$-mediated phosphorylation of MYPT and its association with HSP27 in colonic smooth muscle cells. Am J Physiol Gastrointest Liver Physiol 2006;290:G83G95.

17. Li F, De Godoy M, Rattan S. Role of adenylate and guanylate cyclases in $\beta 1-, \beta 2$, and $\beta 3$-adrenoceptor-mediated relaxation of internal anal sphincter smooth muscle. J Pharmacol Exp Ther 2004;308:1111-1120.

18. Dennis RG, Kosnik PE II. Excitability and isometric contractile properties of mammalian skeletal muscle constructs engineered in vitro. In Vitro Cell Dev Biol Anim 2000;36:327-335.

19. Dennis RG, Kosnik PE. Mesenchymal cell culture: instrumentation and methods for evaluating engineered muscle. In: Atala A, Lanza R, eds. Methods in tissue engineering. San Diego: Harcourt, Academic Press, 2002:9.

20. Hecker L, Baar K, Dennis RG, et al. Development of a threedimensional physiological model of the internal anal sphincter bioengineered in vitro from isolated smooth muscle cells. Am J Physiol Gastrointest Liver Physiol 2005;289:G188-G196.

21. Macpherson PC, Dennis RG, Faulkner JA. Sarcomere dynamics and contraction-induced injury to maximally activated single muscle fibres from soleus muscles of rats. J Physiol 1997;500:523533.

22. Biancani P, Walsh J, Behar J. Vasoactive intestinal peptide: a neurotransmitter for relaxation of the rabbit internal anal sphincter. Gastroenterology 1985;89:867-874.

23. Glavind EB, Forman A, Madsen G, et al. Effects of transmural field stimulation in isolated smooth muscle of human rectum and internal anal sphincter. Am J Physiol Gastrointest Liver Physiol 1997;272:G1075-G1082.

24. Martens JR, Navarro-Polanco R, Coppock EA, et al. Differential targeting of Shaker-like potassium channels to lipid rafts. J Biol Chem 2000;275:7443-7446.

25. Rattan S, De Godoy MA, Patel CA. Rho kinase as a novel molecular therapeutic target for hypertensive internal anal sphincter. Gastroenterology 2006;131:108-116.

26. Freshney RI. Culture of animal cells. New York: Wiley-Liss Inc, 1994.

27. Banwait KS, Rattan S. Role of nitric oxide in $\beta 3$-adrenoceptor activation on basal tone of internal anal sphincter. Am J Physiol Gastrointest Liver Physiol 2003;285:G547-G555.

28. Bitar KN, Hillemeier C, Biancani P. Differential regulation of smooth muscle contraction in rabbit internal anal sphincter by substance $P$ and bombesin. Life Sci 1990;47:2429-2434.

29. Glavind EB, Forman A, Madsen G, et al. Mechanical properties of isolated smooth muscle from human rectum and internal anal sphincter. Am J Physiol 1993;265:G792-G798.

30. Knudsen MA, Glavind EB, Tottrup A. Transmitter interactions in rabbit internal anal sphincter. Am J Physiol 1995;269:G232G239.
31. Ross JJ, Tranquillo RT. ECM gene expression correlates with in vitro tissue growth and development in fibrin gel remodeled by neonatal smooth muscle cells. Matrix Biol 2003;22:477-490.

32. Huang YC, Dennis RG, Baar K. Development of a novel method for engineering 3-dimensional muscle for long-term culture of skeletal muscle. (Abstract) In: Experimental biology. Washington, DC: 2004.

33. Kanada K, Matsuda T. Mechanical stress-induced orientation and ultrastructural change of smooth muscle cells cultured in three-dimensional collagen lattices. Cell Transplant 1994;3: 481-492.

34. Kim BS, Mooney DJ. Scaffolds for engineering smooth muscle under cyclic mechanical strain conditions. J Biomech Eng 2000; 122:210-215.

35. Lee AA, Graham DA, Dela Cruz S, et al. Fluid shear stress-induced alignment of cultured vascular smooth muscle cells. J Biomech Eng 2002;124:37-43.

36. Stegemann J, Nerem RM. Effect of mechanical stimulation on smooth muscle cell proliferation and phenotype. Bioeng Conference 2001;50:609-610.

37. Rembold CM, Foster DB, Strauss JD, et al. cGMP-mediated phosphorylation of heat shock protein 20 may cause smooth muscle relaxation without myosin light chain dephosphorylation in swine carotid artery. J Physiol 2000;524:865-878.

38. GrassI ED, Oegema TR, Tranquillo RT. A fibrin-based arterial media equivalent. J Biomed Mater Res 2003;66A:550-561.

39. Janis RA, Diamond J. Relationship between cyclic nucleotide levels and drug-induced relaxation of smooth muscle. J Pharmacol Exp Ther 1979;211:480-484.

40. Moummi C, Rattan S. Effect of methylene blue and N-ethylmaleimide on internal anal sphincter relaxation. Am J Physiol 1988; 255:G571-G578.

41. Napoli JL, Mellon WS, Fivizzani MA, et al. Direct chemical synthesis of $1 \alpha 25$-dihydroxy[26,27-3H]vitamin D3 with high specific activity: its use in receptor studies. Biochemistry 1980;19: 2515-2521.

Received August 20, 2008. Accepted March 17, 2009.

\section{Reprint requests}

Address requests for reprints to: Khalil N. Bitar, PhD, AGAF, University of Michigan Medical School, 1150 W. Medical Center Dr., MSRB I, Room A520, Ann Arbor, Michigan 48109-0658. e-mail: bitar@umich.edu; fax: (734) 647-9703.

\section{Acknowledgments}

The authors thank Amin Farokhrani, MSc, Engineering, and Shreya Raghavan, MSc, Engineering, for technical assistance.

Conflicts of interest

The authors disclose no conflicts.

Funding

Supported by National Institutes of Health Grant NIDDK R01-DK071614. 